\title{
Is Non-Alcoholic Fatty Liver Disease Less Frequent among Women with Prader-Willi Syndrome?
}

\author{
Giorgio Bedognia, b Graziano Grugnic Valerio Nobilid Fiorenza Agosti ${ }^{\mathrm{e}}$ \\ Antonella Saezzac Alessandro Sartorioc, e \\ ${ }^{a}$ Liver Research Center, Basovizza, Trieste, ${ }^{b}$ International Center for the Assessment of \\ Nutritional Status (ICANS), University of Milan, Milan, ' $D i v i s i o n$ of Auxology, Istituto \\ Auxologico Italiano, IRCCS, Piancavallo, Verbania, 'Liver Research Unit, Bambino Gesù \\ Children's Hospital and Research Institute, Rome, experimental Laboratory for Auxo- \\ Endocrinological Research, Istituto Auxologico Italiano, IRCCS, Milan, Italy
}

\section{Key Words}

Prader-Willi syndrome · Obesity · Body composition · Dual energy X-ray absorptiometry · Insulin resistance

\begin{abstract}
Objective: Patients with Prader-Willi syndrome (PWS) have been hypothesized to be at lower risk of non-alcoholic fatty liver disease (NAFLD) because of higher insulin sensitivity. However, PWS patients have a peculiar body composition, i.e. higher fat mass and lower fat-free mass, which may confound such associations. We evaluated whether NAFLD is less frequent in PWS than in non-PWS women matched on percent body fat (PBF). Methods: PBF was measured by dual-energy X-ray absorptiometry. Liver fat was assessed by ultrasonography. Insulin sensitivity and beta-cell function were evaluated by oral glucose tolerance testing. Coarsened exact matching (CEM) was used to match PWS and non-PWS women on PBF. General and generalized linear models taking CEM into account were used to perform comparisons between PWS and non-PWS women. Results: 20 women with PWS were matched to 27 women without PWS on the basis of PBF (mean 53 vs. $54 \%, p=0.6$ ). Insulin sensitivity and beta-cell function were similar in the two groups. However, the prevalence of NAFLD was $25 \%$ in PWS versus $59 \%$ in non-PWS women $(p=0.04)$. Conclusion: NAFLD is less frequent in PWS than in non-PWS women but this finding is not associated with higher insulin sensitivity.
\end{abstract}


Bedogni et al.: Is Non-Alcoholic Fatty Liver Disease Less Frequent among Women with Prader-Willi Syndrome?

\section{Introduction}

Prader-Willi syndrome (PWS) is a genetic disorder due to absent expression of the paternally active genes in the PWS critical region of chromosome 15 [1, 2]. PWS has an estimated incidence rate of 1 in 25,000 live births and its major clinical features are muscular hypotonia, childhood-onset obesity, multiple endocrinopathies, sleep disorders, developmental delay with cognitive impairment, and characteristic appearance $[3,4]$.

Because of hyperphagia, PWS patients gain substantial weight between 1 and 6 years of age and most of them develop morbid obesity [3]. PWS patients do not only have a higher fat mass (FM) but also a lower fat-free mass (FFM) and resting energy expenditure (REE) as compared to obese non-PWS patients [5-8]. The fact that the REE:FFM ratio of PWS subjects is similar to that of non-PWS subjects suggests that a decrease in FFM, rather than an anomaly of cell metabolism, is responsible for the lower REE [6].

On the basis of body composition alone, one would predict a lower insulin sensitivity in PWS patients because of their lower FFM and higher FM $[9,10]$. However, there is some evidence that PWS subjects are more insulin-sensitive than non-PWS subjects [11]. Mostly on the basis of such higher insulin sensitivity, PWS subjects have been hypothesized to be at lower risk of non-alcoholic fatty liver disease (NAFLD) [11]. These findings may be partly due to the fact that BMI has been used as a measure of body fatness in most studies while, for the reasons discussed above, BMI is not a good index of body composition in PWS patients.

The aim of the present cross-sectional study was to compare the degree of insulin sensitivity and the prevalence of NAFLD in PWS and non-PWS women matched with a direct measure of percent body fat (PBF) obtained by dual-energy X-ray absorptiometry (DXA).

\section{Subjects and Methods}

Subjects

20 obese women with PWS were consecutively studied at the Division of Auxology of the Istituto Auxologico Italiano (Piancavallo, Italy) between January 2010 and January 2012. All patients showed the typical PWS phenotype [3]. 14 women had interstitial deletion of the proximal long arm of chromosome 15 and 6 had uniparental maternal disomy for chromosome 15. PWS women were matched on PBF with 27 women with essential obesity, followed during the same period at the Division of Metabolic Diseases of the Istituto Auxologico Italiano. Exclusion criteria were: age < 18 years, alcohol consumption (any quantity), and hepatitis B virus (HBV) or hepatitis C virus (HCV) infection. The study protocol was approved by the local Ethics Committee, and informed consent was obtained from all subjects.

\section{Clinical and Laboratory Evaluation}

Alcohol consumption was excluded by interview. Weight and height were measured following standard procedures and BMI was calculated as $\mathrm{kg} / \mathrm{m}^{2}$ [12]. Glucose and insulin were measured at $0,30,60,90$, and 120 min during oral glucose tolerance testing (OGTT). Glucose was measured using standard laboratory methods and insulin by using a chemiluminescent immunoassay (Immulite 2000; Diagnostic Products Corporation, Los Angeles, CA, USA). The insulin sensitivity index (ISI) was calculated from OGTT as described by Matsuda and DeFronzo [13]. The ratio between the incremental areas under the curve of insulin and glucose (incAUCins/incAUCglu) was used as marker of beta-cell function [9, 14, 15]. Alanine transaminase (ALT) and gamma-glutamyltransferase (GGT) were measured by standard laboratory methods. HBV or HCV infection was excluded as described elsewhere [16].

\section{Dual-Energy X-Ray Absorptiometry}

FM, lean tissue mass (LTM), and bone mineral content (BMC) were measured using a GE Lunar Prodigy densitometer (GE Lunar Medical Systems, Milwaukee, WI, USA) $[17,18]$. PBF was calculated as FM/(FM + $\mathrm{LTM}+\mathrm{BMC}$ ). In our laboratory, the within-day coefficient of variation for the measurement of PBF is $2 \%$ [18, 19]. 
Bedogni et al.: Is Non-Alcoholic Fatty Liver Disease Less Frequent among Women with Prader-Willi Syndrome?

Indirect Calorimetry

REE was measured using an open-circuit, canopy-equipped indirect calorimeter (Vmax 29; Sensor Medics, Yorba Linda, CA, USA) as described in detail elsewhere [20]. REE was calculated from oxygen uptake and carbon dioxide output using Weir equation [21].

Liver Ultrasonography

An experienced radiologist performed liver ultrasonography using standardized criteria [22]. Normal liver was defined as the absence of liver steatosis and other liver abnormalities. NAFLD was defined as any degree of liver steatosis in the absence of HBV and HCV infection as well as alcohol consumption [23].

Statistical Analysis

Coarsened exact matching (CEM) was used to match PWS and non-PWS women within 5\% of PBF [24]. Continuous measurements of PWS and non-PWS women were compared using a general linear model with PWS status ( $0=$ no; 1 =yes) as predictor, a Gaussian family, an identity link, and robust confidence intervals (CI) [25]. A logarithmic link was used for ISI and incAUCins/incAUCglu to reduce heteroscedasticity of residuals. A generalized linear model with a binomial family, a logarithmic link, and robust CI was used to compare the prevalence of NAFLD in the two study groups using PWS status $(0=$ no; $1=$ yes $)$ as predictor [25]. To test for potential confounding from age, we added age as predictor (continuous, years) to the generalized linear model after having checked that its logit was linear. All models took matching into account by means of CEM-related weights. All statistical tests were two-tailed and statistical significance was set to a value of $\mathrm{p}<0.05$ for all tests. Statistical analysis was performed using Stata 13.0 (Stata Corporation, College Station, TX, USA) together with the user-written cem command [26].

\section{Results}

The measurements of the PWS and non-PWS women are given in table 1.

PWS women were lighter and shorter than non-PWS women, while their percent body composition was similar to that of PWS women because of matching on PBF. As expected, LTM and REE were lower in PWS than in non-PWS women but the REE:LTM ratio was similar.

No between-group differences were found in liver enzymes, fasting, and 120-min glucose, insulin sensitivity as detected by ISI, and beta-cell function as detected by the incAUCins/ incAUCglu ratio.

The prevalence of NAFLD was 59\% (robust 95\% CI 37-80\%) in non-PWS and 25\% $(6-44 \%)$ in PWS women $(\mathrm{p}=0.04)$. Age was not associated with NAFLD both at univariable (odds ratio $(\mathrm{OR})=1.02$ per year of age, $\mathrm{p}=0.5)$ and multivariable analysis $(\mathrm{OR}=0.92, \mathrm{p}=0.9)$, and the percent change of the log-odds of PWS status after inclusion of age into the model was around $1 \%$, showing no evidence of confounding from age.

\section{Discussion}

In this cross-sectional study, we tested whether NAFLD is less frequent in PWS than in non-PWS women. This hypothesis is consistent with the fact that PWS subjects may have a higher insulin sensitivity [11] and that insulin sensitivity is inversely associated with the presence of fatty liver in the general population [27]. We found that NAFLD was less frequent among PWS women and that their insulin sensitivity was similar to that of non-PWS women.

The available studies investigating the relationship between PWS and insulin sensitivity relied mostly on BMI to control for the confounding effect of body fat [11]. However, BMI is just a surrogate measure of body fat, and this is especially true in PWS subjects because of their higher FM and lower FFM as compared to non-PWS subjects [5, 7]. This implies that, at the same level of BMI, PWS and non-PWS subjects will have a different body composition that 
Bedogni et al.: Is Non-Alcoholic Fatty Liver Disease Less Frequent among Women with Prader-Willi Syndrome?

Table 1. Measurements of women with and without PWS matched on PBFa

\begin{tabular}{llll}
\hline & $\begin{array}{l}\text { Non-PWS women } \\
(\mathrm{n}=27)\end{array}$ & $\begin{array}{l}\text { PWS women } \\
(\mathrm{n}=20)\end{array}$ & p value \\
\hline Age, years & $33(30-36)$ & $30(27-32)$ & 0.1 \\
Weight, kg & $111(107-115)$ & $85(78-93)$ & $<0.001$ \\
Height, m & $1.62(1.60-1.65)$ & $1.49(1.46-1.52)$ & $<0.001$ \\
BMI, kg/m ${ }^{2}$ & $42(41-43)$ & $39(35-42)$ & 0.1 \\
FM, kg & $56(52-59)$ & $44(39-49)$ & $<0.001$ \\
FM:BM, \% & $53(51-56)$ & $54(52-56)$ & 0.6 \\
LTM, kg & $47(44-49)$ & $35(32-37)$ & $<0.001$ \\
LTM:BM, \% & $45(43-47)$ & $44(42-46)$ & 0.6 \\
BMC, kg & $2.0(1.8-2.2)$ & $1.5(1.3-1.6)$ & $<0.001$ \\
BMC:BM, \% & $1.9(1.8-2.1)$ & $1.9(1.7-2.2)$ & 0.9 \\
REE, kcal/day & $1,780(1,677-1,882)$ & $1418(1,272-1,564)$ & $<0.001$ \\
REE:LTM, kcal/day/kg & $39(37-41)$ & $41(38-44)$ & 0.2 \\
ALT, U/l & $20(16-24)$ & $25(19-30)$ & 0.2 \\
GGT, U/l & $20(12-28)$ & $23(15-31)$ & 0.6 \\
Glucose, mg/dl & $86(83-89)$ & $83(79-87)$ & 0.3 \\
Glucose 120 min, mg/dl & $125(110-141)$ & $130(117-144)$ & 0.6 \\
ISI & $7(5-9)$ & $8(5-10)$ & 0.7 \\
incAUCins/incAUCglu & $0.97(0.78-1.17)$ & $0.82(0.63-1.01)$ & 0.3 \\
\hline
\end{tabular}

${ }^{a}$ Values are means and robust $95 \%$ confidence intervals.

bVariable on which coarsened exact matching of non-PWS and PWS women was performed.

PWS = Prader-Willi syndrome; PBF = percent body fat; FM = fat mass by dual-energy X-ray absorptiometry (DXA); BM = body mass by DXA; LTM = lean tissue mass by DXA; BMC = bone mineral content by DXA; $\mathrm{REE}$ = resting energy expenditure by indirect calorimetry; ALT = alanine transaminase; GGT = gammaglutamyltransferase; ISI = insulin sensitivity index; inc-AUCins/incAUCglu = ratio between the incremental areas under the curve of insulin and glucose.

may confound between-group comparisons of outcomes that are sensitive to body fat. For instance, the mean difference in BMI between our PWS and non-PWS women was a not negligible $3 \mathrm{~kg} / \mathrm{m}^{2}$ as compared to a clearly negligible one of $1 \%$ for PBF. Thus, it is important that a comparison between PWS and non-PWS subjects for outcomes that are (expected to be) associated with body fat employs more direct methods for measuring body fat [9]. We chose DXA to measure body fat because it effectively and reproducibly ranks the amount of fat in the human body [19].

Our PWS and non-PWS women matched on PBF had a similar insulin sensitivity as detected by OGTT. Although this was not the aim of this study as it is not biologically plausible to match PWS and non-PWS subjects on BMI if the aim is to control for body fat, we tested whether matching on BMI produced different results. After matching on BMI, PWS women appeared to have a higher insulin sensitivity than non-PWS women but still had a lower prevalence of NAFLD (data not shown). This finding gives at least partial support to the possibility that matching on BMI is not enough to control for the confounding effect of body fat. (We would like to add in passing that the matching based on BMI was much less efficient on statistical grounds because more subjects were outside the so-called region of common support [24].)

The prevalence of NAFLD was 25\% in PWS versus 59\% in non-PWS women matched on PBF $(p=0.04)$. We expected that this finding would be accompanied by lower insulin sensitivity but this was not the case. A lower insulin sensitivity is common in NAFLD, even if it is not necessarily a pathogenetic factor or the only pathogenetic factor involved in this disease 
Bedogni et al.: Is Non-Alcoholic Fatty Liver Disease Less Frequent among Women with Prader-Willi Syndrome?

[28]. However, it remains to be explained why PWS patients have a lower prevalence of NAFLD than non-PWS patients matched on PBF.

PWS patients often undergo treatment with growth hormone (GH), which is expected on average to decrease PBF and to increase FFM [29]. More importantly for the aim of this study, GH treatment may reduce liver fat in GH-deficient subjects. 9 of our 20 PWS women had undergone or were undergoing treatment with recombinant GH at different doses and for different times. This number of subjects is clearly too low and, together with the crosssectional nature of this study, does not allow testing any hypothesis about a potential association between GH treatment and NAFLD. However, there is no doubt that such an association should be taken into account in further studies investigating the relationship between PWS and NAFLD.

This study has some limitations. Firstly, it was performed among Caucasian PWS women followed at a tertiary care center, and its results might not be generalizable to non-Caucasian ethnic groups, men, and the general population. In this respect, it is known that the manifestations of PWS may differ according to the ethnicity of the patients and that the prevalence of NAFLD is higher in some ethnic groups, e.g. African-Americans [30, 31]. Men are currently considered to be at a greater risk of NAFLD than women [31], although no sex-based comparison is available for PWS. Secondly, our estimate of the prevalence of NAFLD in PWS women (25\%; 95\% CI 6-44\%) was imprecise because of the relatively low sample size. Taking our point estimate of $25 \%$ for the prevalence of NAFLD as a guide, 60 PWS subjects are needed to detect such a prevalence with a precision ranging from 15 to $38 \%$ (95\% exact $\mathrm{CI}$ ), which is acceptable on clinical grounds. Such a number is unlikely to be reached by a single-center study but it could be obtained by multi-center studies.

In conclusion, NAFLD is less frequent in PWS than in non-PWS women matched on PBF; however, this finding is not associated with lower insulin sensitivity. Multi-center studies are needed to replicate these findings by also taking men and non-Caucasian ethnic groups into account.

\section{Acknowledgements}

The study was supported by Progetti di Ricerca Corrente, Istituto Auxologico Italiano, Verbania, Italy.

\section{Disclosure Statement}

All of the authors declare no conflict of interest.

\section{References}

1 Bittel DC, Butler MG: Prader-Willi syndrome: clinical genetics, cytogenetics and molecular biology. Expert Rev Mol Med 2005;7:1-20.

2 Prader A, Labhart A, Willi H: Ein Syndrom von Adipositas, Kleinwuchs Kryptorchismus und Oligophenie nach myatonieartigem Zustand im Neugeborenalter. Schweiz Med Wschr 1956;86:1260-1261.

-3 Cassidy SB, Schwartz S, Miller JL, Driscoll DJ: Prader-Willi syndrome. Genet Med 2012;14:10-26.

-4 Whittington JE, Holland AJ, Webb T, Butler J, Clarke D, Boer H: Population prevalence and estimated birth incidence and mortality rate for people with Prader-Willi syndrome in one UK Health Region. J Med Genet 2001; 38:792-798.

5 Brambilla P, Bosio L, Manzoni P, Pietrobelli A, Beccaria L, Chiumello G: Peculiar body composition in patients with Prader-Labhart-Willi syndrome. Am J Clin Nutr 1997;65:1369-1374.

6 Schoeller DA, Levitsky LL, Bandini LG, Dietz WW, Walczak A: Energy expenditure and body composition in Prader-Willi syndrome. Metabolism 1988;37:115-120. 
Bedogni et al.: Is Non-Alcoholic Fatty Liver Disease Less Frequent among Women with Prader-Willi Syndrome?

7 Theodoro MF, Talebizadeh Z, Butler MG: Body composition and fatness patterns in Prader-Willi syndrome: comparison with simple obesity. Obesity (Silver Spring) 2006;14:1685-1690.

8 van Mil EA, Westerterp KR, Gerver WJ, Curfs LM, Schrander-Stumpel CT, Kester AD, Saris WH: Energy expenditure at rest and during sleep in children with Prader-Willi syndrome is explained by body composition. Am J Clin Nutr 2000;71:752-756.

-9 Bedogni G, Gastaldelli A, Agosti F, De Col A, Marazzi N, Mazzilli G, Saezza A, Sartorio A: Impact of percent body fat on oral glucose tolerance testing: a cross-sectional study in 1512 obese children. J Endocrinol Invest 2012; 35:893-896.

10 Reaven GM: Banting lecture 1988. Role of insulin resistance in human disease. Diabetes 1988;37:1595-1607.

11 Haqq AM, Muehlbauer MJ, Newgard CB, Grambow S, Freemark M: The metabolic phenotype of Prader-Willi syndrome (PWS) in childhood: heightened insulin sensitivity relative to body mass index. J Clin Endocrinol Metab 2011;96:E225-E232.

12 Lohman TG, Roche AF, Martorell R: Anthropometric standardization reference manual. Champaign, IL, Human Kinetics Books, 1988.

13 Matsuda M, DeFronzo RA: Insulin sensitivity indices obtained from oral glucose tolerance testing: comparison with the euglycemic insulin clamp. Diabetes Care 1999;22:1462-1470.

14 Bedogni G, Gastaldelli A, Manco M, De Col A, Agosti F, Tiribelli C, Sartorio A: Relationship between fatty liver and glucose metabolism: a cross-sectional study in 571 obese children. Nutr Metab Cardiovasc Dis 2012;22: 120-126.

15 Gastaldelli A, Ferrannini E, Miyazaki Y, Matsuda M, DeFronzo RA, San Antonio metabolism study: Beta-cell dysfunction and glucose intolerance: results from the San Antonio metabolism (SAM) study. Diabetologia 2004;47:31-39.

16 Bedogni G, Miglioli L, Masutti F, Ferri S, Castiglione A, Lenzi M, Crocè LS, Granito A, Tiribelli C, Bellentani S: Natural course of chronic HCV and HBV infection and role of alcohol in the general population: the Dionysos Study. Am J Gastroenterol 2008;103:2248-2253.

-17 Pietrobelli A, Formica C, Wang Z, Heymsfield SB: Dual-energy X-ray absorptiometry body composition model: review of physical concepts. Am J Physiol 1996;271:E941-E951.

18 Bedogni G, Agosti F, De Col A, Marazzi N, Tagliaferri A, Sartorio A: Comparison of dual-energy X-ray absorptiometry, air displacement plethysmography and bioelectrical impedance analysis for the assessment of body composition in morbidly obese women. Eur J Clin Nutr 2013;67:1129-1132.

19 Lazzer S, Bedogni G, Agosti F, De Col A, Mornati D, Sartorio A: Comparison of dual-energy X-ray absorptiometry, air displacement plethysmography and bioelectrical impedance analysis for the assessment of body composition in severely obese Caucasian children and adolescents. Br J Nutr 2008;100:918-924.

20 Lazzer S, Bedogni G, Lafortuna CL, Marazzi N, Busti C, Galli R, De Col A, Agosti F, Sartorio A: Relationship between basal metabolic rate, gender, age, and body composition in 8,780 white obese subjects. Obesity (Silver Spring) 2010;18:71-78.

-21 Weir JB: New methods for calculating metabolic rate with special reference to protein metabolism. 1949. Nutrition 1990;6:213-221.

-22 Shannon A, Alkhouri N, Carter-Kent C, Monti L, Devito R, Lopez R, Feldstein AE, Nobili V: Ultrasonographic quantitative estimation of hepatic steatosis in children with NAFLD. J Pediatr Gastroenterol Nutr 2011;53: 190-195.

23 Sartorio A, Del Col A, Agosti F, Mazzilli G, Bellentani S, Tiribelli C, Bedogni G: Predictors of non-alcoholic fatty liver disease in obese children. Eur J Clin Nutr 2007;61:877-883.

24 Iacus SM, King G, Porro G: Multivariate matching methods that are monotonic imbalance bounding. J Am Stat Assoc 2011;106:345-361.

25 Hardin JW, Hilbe JM: Generalized linear models and extensions. College Station, TX, Stata Press, 2012.

26 Blackwell M, Iacus S, King G, Porro G: cem: coarsened exact matching in Stata. Stata J 2009; 9:524-546.

-27 Bedogni G, Bellentani S, Miglioli L, Masutti F, Passalacqua M, Castiglione A, Tiribelli C: The Fatty Liver Index: a simple and accurate predictor of hepatic steatosis in the general population. BMC Gastroenterol 2006;6:33.

-28 Nobili V, Bedogni G, Berni Canani R, Brambilla P, Cianfarani S, Pietrobelli A, Agostoni C: The potential role of fatty liver in paediatric metabolic syndrome: a distinct phenotype with high metabolic risk? Pediatr Obes 2012;7:e75-e80.

29 Sanchez-Ortiga R, Klibanski A, Tritos NA: Effects of recombinant human growth hormone therapy in adults with Prader-Willi syndrome: a meta-analysis. Clin Endocrinol (Oxf) 2012;77:86-93.

30 Hudgins L, Geer JS, Cassidy SB: Phenotypic differences in African Americans with Prader-Willi syndrome. Genet Med 1998;1:49-51.

31 Ratziu V, Bellentani S, Cortez-Pinto H, Day C, Marchesini G: A position statement on NAFLD/NASH based on the EASL 2009 special conference. J Hepatol 2010;53:372-384. 